\title{
Aprendizagem utilizando Dispositivos Móveis com Sistemas de Realidade Virtual
}

\author{
Edgar Marçal ${ }^{*}$ \\ Rossana Andrade** \\ Riverson Rios ${ }^{* *}$
}

\begin{abstract}
Resumo. A computação móvel emerge como uma tecnologia inovadora para a área educacional. Neste trabalho é apresentado um framework para a construção de aplicações educacionais em dispositivos móveis com recursos de realidade virtual. Este framework tem como objetivo principal fornecer ao desenvolvedor uma arquitetura consistente para implementação de programas em dispositivos móveis, com foco na aprendizagem. Para validação do framework foi desenvolvido um protótipo que demonstra sua interatividade, usabilidade e portabilidade, além de permitir uma experimentação da integração das tecnologias de realidade virtual e computação móvel.

Palavras-Chave: Aprendizagem Móvel, Realidade Virtual, Framework
\end{abstract}

\begin{abstract}
Mobile computing emerges as an innovative technology for the educational area. In this work framework for the construction of educational applications in mobile devices with resources of virtual reality is presented one. This framework has as objective main to supply to the desenvolvedor a consistent architecture implementation of programs in mobile devices, with focus in the learning. For validation of framework an prototype that demonstrates its interatividade, usability and portabilidade, besides allowing to an experimentation of the integration of the technologies of virtual reality and mobile computation was developed.
\end{abstract}

Keywords: Mobile Learning, Virtual Reality, Framework

\section{Introdução}

As tecnologias de computação móvel encontram-se atualmente em franca evolução e parecem destinadas a transformar-se no novo paradigma dominante da computação (Myers et al., 2003). A utilização de dispositivos móveis na educação criou um novo conceito, o chamado Mobile Learning ou m-Learning. Seu grande potencial encontra-se na utilização da tecnologia móvel como parte de um modelo de aprendizado integrado, caracterizado pelo uso de dispositivos de comunicação sem fio, de forma transparente e com alto grau de mobilidade (Ahonen, 2003; Syvänen, 2003). Essa oferta de serviços de telecomunicações e de artefatos computacionais, capazes de prover mobilidade aos diferentes participantes de projetos educacionais, apresenta a oportunidade para o desenvolvimento de pesquisas no campo da computação móvel aplicadas à educação (Meirelles et al., 2004).

\footnotetext{
* Mestrando em Ciência da Computação - Universidade Federal do Ceará (UFC) edgar@lia.ufc.br

** Professor(a) Doutor(a) do Mestrado e Doutorado em Ciência da Computação da UFC \{rossana, riverson\}@ufc.br
} 
apoio à aprendizagem, os mundos tridimensionais virtuais, onde o sujeito possa se movimentar, ouvir, ver e manipular objetos, como no mundo real, representam interessantes oportunidades à disposição dos educadores. A introdução dos chamados Sistemas de Realidade Virtual (SRV) na educação pode modificar significativamente o papel destes educadores (Ferreira et al., 2004).

Os recursos de realidade virtual nas aplicações de m-Learning podem propiciar um incremento na aprendizagem ao fornecerem os seguintes benefícios:

- Apoiar e motivar o aprendizado em excursões, fornecendo cenários virtuais semelhantes aos reais acrescidos de informações complementares (Bricken, 1993).

- Melhorar a compreensão sobre determinada obra ou experimento, através de uma maior aproximação e de uma visualização sob diferentes ângulos (Erickson, 1993).

- Permitir a simulação e a análise de experiências recém vivenciadas, seja na própria sala de aula, no laboratório ou em passeios educativos.

- Possibilitar a demonstração do funcionamento de equipamentos, através de simulações, para auxiliar na resolução de problemas técnicos no momento do atendimento. Funcionando como um manual tridimensional (3D) portátil.

Inúmeros desafios se apresentam quando são projetadas aplicações educacionais para dispositivos móveis com características de realidade virtual: Como produzir um cenário 3D motivador, juntamente com imagens e informações nas reduzidas telas dos telefones celulares? Como possibilitar formas de interação eficientes? Como contornar a limitação de memória e processamento dos dispositivos móveis?

Este trabalho apresenta o VirTraM (Virtual Training for Mobile Devices), um framework orientado a objetos voltado para o desenvolvimento de aplicações educacionais inovadoras, que incorporam recursos de realidade virtual em dispositivos móveis. Este framework pretende solucionar os desafios mencionados anteriormente, tornando-se uma solução de arquitetura para a construção de aplicações educacionais em dispositivos móveis.

Este artigo apresenta, na Seção 2, aspectos envolvidos na aprendizagem através da utilização de dispositivos móveis e características de SRVs aplicados à educação. A Seção 3 apresenta a arquitetura do framework, destacando os requisitos das aplicações. A Seção 5 descreve o estudo de caso implementado. E a Seção 6 apresenta as conclusões e os trabalhos futuros.

\section{Educação: Computação Móvel e Realidade Virtual}

Este artigo fundamenta-se na utilização de dispositivos móveis para a aprendizagem, caracterizando a área de m-Learning descrita na Seção 1, utilizando Sistemas de Realidade Virtual.

\subsection{Mobile Learning}

O paradigma Mobile Learning ou m-Learning surge aproveitando-se da disponibilidade de dispositivos móveis e considerando-se as necessidades específicas de educação e treinamento (Nyiri, 2002).

As pesquisas em m-Learning têm-se voltado para dois grupos de usuários principais: crianças e profissionais que exercem suas atividades em campo, neste artigo 
definidos como trabalhadores externos. Dispositivos móveis fornecem um novo e motivador paradigma de interação, particularmente para as crianças, e várias iniciativas têm sido desenvolvidas nessa área (Oosterholt, 1996; Danesh, 2001). No caso de trabalhadores externos, cuja rotina é bastante dinâmica, envolvendo viagens a diferentes localidades, a preocupação é fornecer um ambiente de aprendizado que ponha sempre à sua disposição a informação mais atualizada possível. Desta forma, o m-Learning surge como uma importante alternativa de ensino e treinamento à distância, na qual podem ser destacados os seguintes objetivos:

- Melhorar os recursos para o aprendizado do aluno, que poderá contar com um dispositivo computacional para execução de tarefas, anotação de idéias, consulta de informações via Internet, registro de fatos através de câmera digital, gravação de sons e outras funcionalidades existentes;

- Prover acesso aos conteúdos didáticos em qualquer lugar e a qualquer momento, de acordo com a conectividade do dispositivo;

- Aumentar as possibilidades de acesso ao conteúdo, incrementando e incentivando a utilização dos serviços providos pela instituição, educacional ou empresarial;

- Expandir o corpo de professores e as estratégias de aprendizado disponíveis, através de novas tecnologias que dão suporte tanto à aprendizagem formal como à informal;

- Fornecer meios para o desenvolvimento de métodos inovadores de ensino e de treinamento, utilizando os novos recursos de computação e de mobilidade.

O instituto de pesquisa SRI (Stanford Research Institute) realizou uma pesquisa sobre a utilização de dispositivos móveis nas escolas (Crawford et al., 2002). Foram pesquisadas mais de 100 instituições de ensino nos Estados Unidos, do ano de 2000 a 2002. Este estudo concluiu que os dispositivos móveis podem oferecer benefícios únicos aos alunos, conforme descrito a seguir. Os professores participantes demonstraram uma grande aceitação da computação móvel em suas salas de aula. A seguir são apresentados alguns números que demonstram essa aceitação:

- $89 \%$ dos professores disseram que descobriram nos dispositivos móveis eficientes ferramentas de ensino;

- $93 \%$ dos professores acreditam que os dispositivos móveis podem ter um impacto positivo na aprendizagem dos alunos;

- $90 \%$ dos professores pretendem continuar a utilizar os dispositivos móveis em suas aulas;

- $75 \%$ dos professores que permitiram que os alunos levassem os dispositivos móveis para casa, constataram um aumento na conclusão dos trabalhos de casa;

- Entre os alunos, 66\% acharam confortável o uso do dispositivo móvel.

- Quase a totalidade dos professores afirmou que a utilização de softwares educativos apropriados e acessórios foi de fundamental importância na aprendizagem, ao complementar os recursos básicos dos dispositivos móveis. Por exemplo: alunos podem utilizar sondas acopladas a PDAs para medir e registrar a qualidade da água dos rios. 
Em geral, a maioria dos professores afirmou que a introdução da computação móvel na sala de aula aumentou a motivação para aprender, a colaboração e a comunicação entre os estudantes.

Em particular, dispositivos de comunicação sem fio oferecem a uma extensão natural da educação a distância via computadores (Lehner et al. , 2002), pois contribuem para a facilidade de acesso ao aprendizado, por exemplo, na obtenção de conteúdo específico para um determinado assunto, sem hora e local pré-estabelecidos.

\subsection{A Realidade Virtual no Contexto Educacional}

Kirner (2004) define realidade virtual (RV) como uma técnica avançada de interface, onde o usuário pode realizar imersão, navegação e interação em um ambiente sintético tridimensional gerado por computador, utilizando canais multi-sensoriais. Através de cenários tridimensionais, é possível representar uma grande variedade de situações voltadas para diversas áreas de aplicação, tais como: excursões virtuais em mundos reais (como museus, terrenos, etc.) ou imaginários e representação de objetos (carros, máquinas, etc) ou personificações de seres reais (homem, animal, etc) ou imaginários (alienígenas).

Como um ambiente de apoio à aprendizagem, os SRVs disponibilizam aos educadores, a oportunidade de possibilitar aos alunos o aprendizado por experimentação, pois o aluno poderá movimentar-se, ouvir, ver e manipular objetos, como se estivesse no mundo real. A Tabela 1 apresenta algumas áreas potenciais onde os benefícios dos SRVs educacionais podem ser esperados.

Tabela 1: Uso potencial dos SRVs (Ferreira et al., 2004)

\begin{tabular}{|l|l|}
\hline Uso potencial & Benefícios comparados com os métodos tradicionais \\
\hline Simulação de sistemas complexos & $\begin{array}{l}\text { Habilidade para observar a operação do sistema a partir } \\
\text { de um número de perspectivas aliadas a uma alta } \\
\text { qualidade de visualização e interação. }\end{array}$ \\
\hline $\begin{array}{l}\text { Visualização macroscópica e } \\
\text { microscópica }\end{array}$ & $\begin{array}{l}\text { Observação de propriedades de objetos, que são muito } \\
\text { grandes ou muito pequenos para serem observados em } \\
\text { escala normais. }\end{array}$ \\
\hline $\begin{array}{l}\text { Simulação em tempos mais } \\
\text { rápidos ou mais lentos }\end{array}$ & $\begin{array}{l}\text { Habilidade para controlar a escala de tempo em um } \\
\text { evento dinâmico. Esta facilidade pode operar como no } \\
\text { avanço ou retrocesso rápido dos gravadores de vídeo } \\
\text { modernos. }\end{array}$ \\
\hline Altos níveis de interatividade & $\begin{array}{l}\text { Os SRVs permitem um grau maior de interatividade que } \\
\text { outros sistemas baseados em computador. }\end{array}$ \\
\hline Sensação de imersão & $\begin{array}{l}\text { Em algumas aplicaçães, a sensação de escala é } \\
\text { extremamente importante. }\end{array}$ \\
\hline Flexibilidade e adaptabilidade & Um mesmo SRV pode ser alocado a diversos usos. \\
\hline
\end{tabular}

\section{VirTraM}

Um framework orientado a objetos é um modelo reutilizável representado por um conjunto de classes que colaboram entre si e constituem a arquitetura principal de uma aplicação ou sub-sistema (Mattsson, 1996). A idéia principal de um framework é 
permitir que um conjunto de recursos comuns seja reaproveitado para cada novo software criado. A utilização de frameworks facilita o desenvolvimento de aplicações ao permitir que o programador trabalhe com menos elementos, abstraindo os detalhes de implementação. Outros benefícios são a modularidade e a re-usabilidade (Fayad et al., 1999).

O VirTraM tem como objetivo principal provê uma arquitetura para a construção de aplicações voltadas ao treinamento de indivíduos utilizando dispositivos móveis com recursos de realidade virtual. As aplicações desenvolvidas a partir do VirTraM atenderão determinados requisitos em comum, descritos a seguir.

Mobilidade. O usuário está em movimento e a aplicação deve poder ser usada em qualquer local e a qualquer momento, sempre que for preciso. Desta forma, não pode haver dependência dos recursos de comunicação. Antes da instalação, é necessário copiar a aplicação, que se encontra em um servidor, para o dispositivo móvel, que funciona como cliente. Após ser instalada, a aplicação deve funcionar localmente no dispositivo e independentemente de uma conexão de dados, com ou sem fio. Assim, garante-se que a aprendizagem não será interrompida em caso de ausência de comunicação.

Interatividade. Segundo Rossou (2004) a interatividade é a razão de ser de um mundo virtual. Nesse contexto, a interatividade está associada à habilidade do usuário escolher um caminho dentro do mundo virtual, podendo navegar livremente e manipular os objetos tridimensionais. As formas de manipulação podem ser as operações de rotação e translação e o disparo das animações específicas dos objetos.

Portabilidade (Heterogeneidade). A aplicação pode ser executada em diferentes plataformas, característica presente tanto na computação móvel como na realidade virtual (Luchini, 2004; Marson, 2003). A aplicação deve ser compatível tanto com telefones celulares quanto com PDAs, suportando diferentes sistemas operacionais.

Facilidade de uso. Segundo Avellis et al. (2003), a aplicação deve ser simples e fácil de usar, pois o usuário não tem como atividade fim a utilização da aplicação móvel, e sim através dela atingir uma determinada finalidade. Isso é importante principalmente quando se é levada em consideração a complexidade de aplicações com recursos de realidade virtual.As seguintes técnicas são empregadas para proporcionar uma maior facilidade de uso da aplicação móvel:

- Minimizar a entrada de texto;

- Evitar muitos níveis de profundidade nas telas da aplicação, ou seja, uma tela que chama outra tela que por sua vez chama outra tela e assim por diante;

- Utilizar imagens para diminuir a quantidade de texto.

Informação essencial. As tecnologias de computação móvel devem ser utilizadas em função das tarefas a realizar, mantendo a informação essencial e descartando a informação supérflua.

Conteúdo. A aplicação deve possuir um repositório de informações para possibilitar ao usuário um melhor entendimento do ambiente virtual. Através de representação tridimensional, simulação de fenômenos do mundo real, informações descritivas e imagens, o usuário tem uma melhor compreensão do treinamento. 


\subsection{Arquitetura}

O VirTraM é composto por um conjunto de classes que serão adaptadas pelo programador para o desenvolvimento de uma aplicação educacional específica, que atenderá os requisitos mencionados no início da Seção 3. Para a implementação do VirTraM utilizou-se a plataforma Java 2 para dispositivos móveis (J2ME - Java 2 Micro Edition) (Sun, 2004). Adotou-se esse ambiente principalmente pela sua grande portabilidade (suportado pela maioria dos fabricantes de dispositivos móveis). As classes do VirTraM colaboram entre si e devem ser reutilizadas como um todo para a construção da aplicação. O VirTraM encapsula os detalhes de implementação do J2ME e permite ao programador concentrar-se nos aspectos da aplicação de treinamento.

A Figura 1 apresenta a arquitetura do VirTraM, cujos detalhes são descritos a seguir.

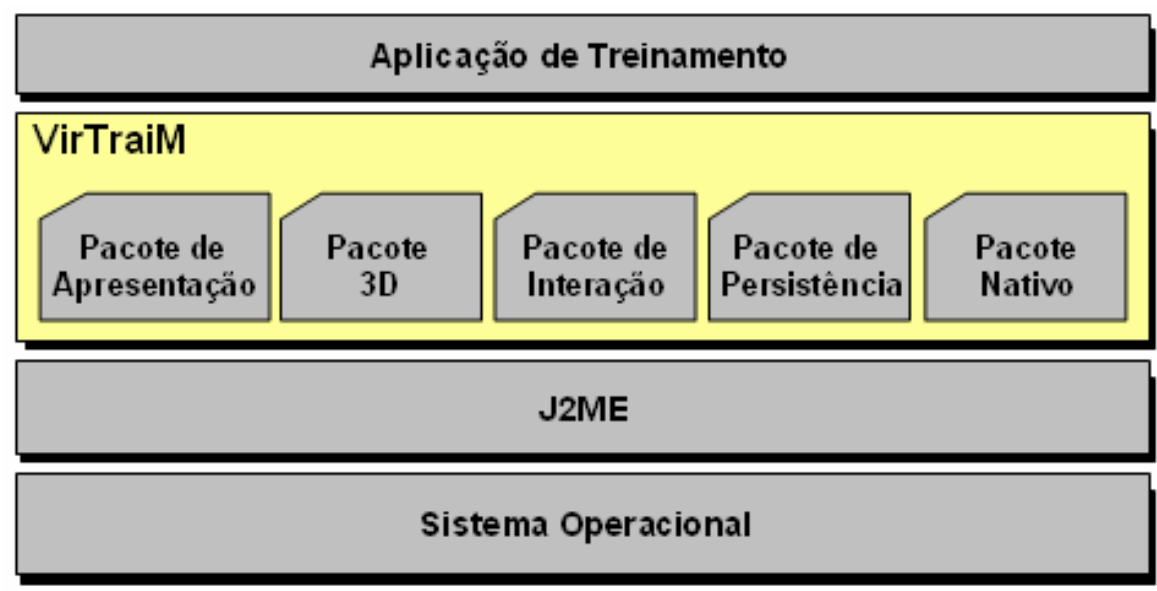

Figura 1. Arquitetura do VirTraM

O Pacote de Apresentação consiste de conjunto de classes responsáveis pelas funcionalidades visuais utilizadas nas interfaces da aplicação, tais como: menu com as interações, avisos de alertas e caixas de seleção.

O Pacote 3D contém as classes essenciais para a construção dos ambientes tridimensionais das aplicações. Responsáveis pelas funções $3 \mathrm{D}$, tais como: renderização, rotação, translação, mudança do ângulo de visão virtual e detecção de colisão. Um outro recurso importante desse pacote é o apontador, utilizado no ambiente para selecionar os objetos 3D.

O Pacote de Interação é responsável por capturar as diferentes interações do usuário, disparar as funcionalidades solicitadas e retornar os resultados para a aplicação. As formas de interação previstas são o teclado ou a tela sensível ao toque do dispositivo móvel. Porém, o framework pode ser estendido para suportar outras formas de interação, como localização via GPS ou Wi-Fi.

O Pacote de Persistência fornece as classes responsáveis pelo acesso aos dados da aplicação. Entre as principais funcionalidades desse pacote podemos destacar consulta, armazenamento e alteração das informações do treinamento.

O Pacote Nativo fornece as classes responsáveis pelo acesso aos recursos específicos de determinados dispositivos, tais como: recursos de som e função para vibrar o dispositivo móvel. 


\section{Estudo de Caso}

O museu virtual foi escolhido como estudo de caso para o VirTraM. Algumas iniciativas têm sido desenvolvidas com o objetivo de representar museus do mundo real em computadores (Wojciechowski, 2004; Corcoran, 2002; Charitos, 2001; Wazlawick, 2001). Porém, é importante salientar que a maioria dos museus encontrados na Internet, ditos virtuais, trata-se apenas de simples páginas Web, sem recursos de realidade virtual. Desta forma, este trabalho utiliza o termo museu virtual para ambientes tridimensionais interativos que possibilitam navegação, simulação e sensação de imersão, total ou parcial. Algumas das vantagens dos museus virtuais são: motivação para uma maior exploração do acervo do museu, através da possibilidade de aproximação e manipulação das obras; visualização e simulação de experimentos nos museus de ciências, para que os alunos possam fixar os conceitos teóricos aprendidos; e, maior poder de ilustração já que a realidade virtual, possibilitando a visão em três dimensões, permite o emprego de múltiplas camadas de referência (profundidade, tamanho, posição, etc.) no aprendizado.

O protótipo implementado utiliza recursos de realidade virtual para simular um museu real ou imaginário, propiciando excursões virtuais interativas e aumentando a motivação para a aprendizagem. Dispositivos móveis poderão ser utilizados no interior dos museus, funcionando como guias virtuais e fornecendo informações importantes sobre as obras durante as visitas.

O protótipo consiste de um programa para dispositivos móveis, implementado a partir do VirTraM e que possibilita a obtenção de conhecimento sobre os acervos de museus. $\quad \mathrm{O}$ desenvolvimento não tomou como base um museu real. $\mathrm{O}$ ambiente tridimensional é composto por duas salas, com quadros e uma escultura. $\mathrm{O}$ visitante pode navegar pelas salas, olhando para os lados, para cima e para baixo. Toda a interação é feita com a utilização das teclas do telefone celular. $\mathrm{O}$ usuário pode, ora movimentar-se, ora movimentar o apontador, ou ainda movimentar um objeto 3D. Ao selecionar um objeto 3D, um menu aparece e o usuário pode escolher entre consultar a descrição daquela obra ou executar uma animação associada, caso exista. É importante ressaltar que o protótipo implementado utiliza o conceito de realidade virtual não imersiva, a qual não necessita de equipamentos sofisticados como luvas 3D ou óculos especiais. A Figura 2 apresenta algumas telas do museu virtual desenvolvido, capturadas a partir do emulador do Wireless Toolkit (WTK) (Sun, 2004). 
a)

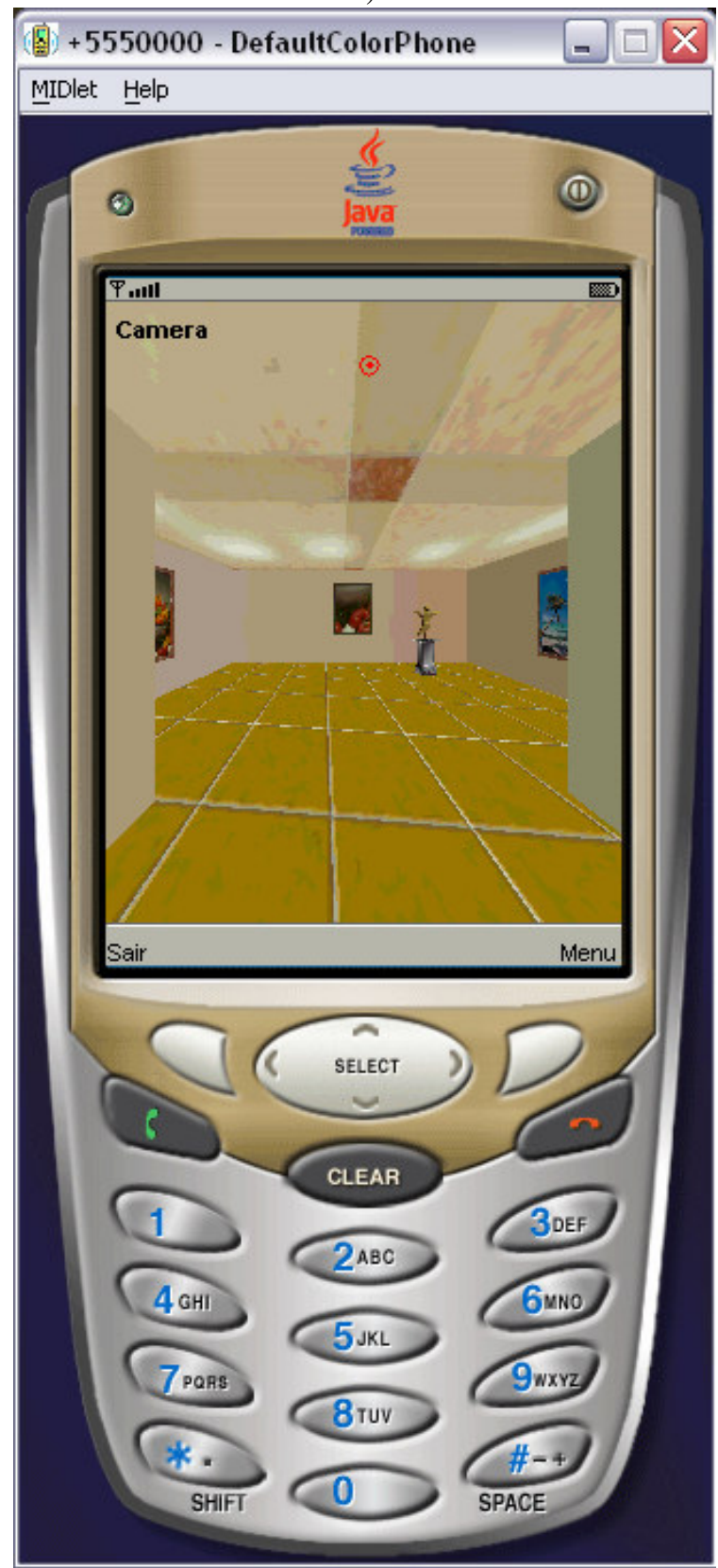

b)

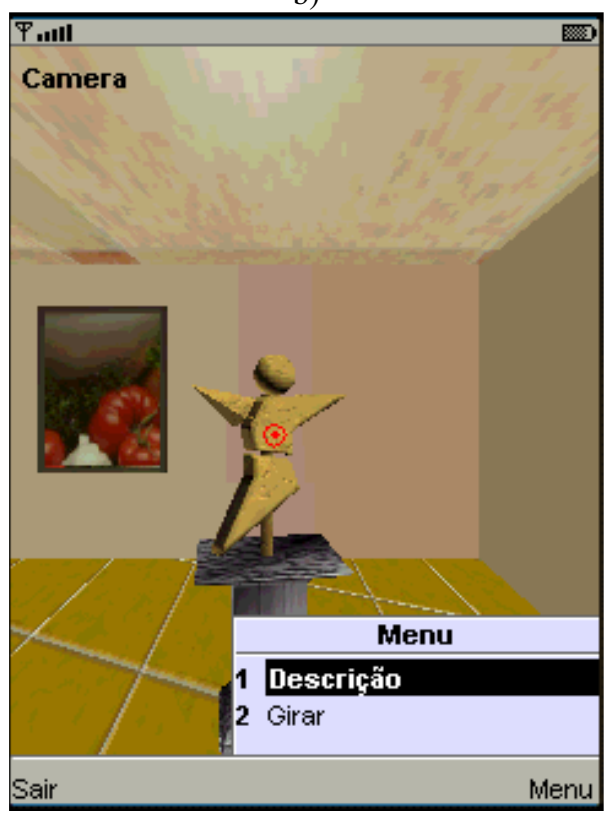

c)

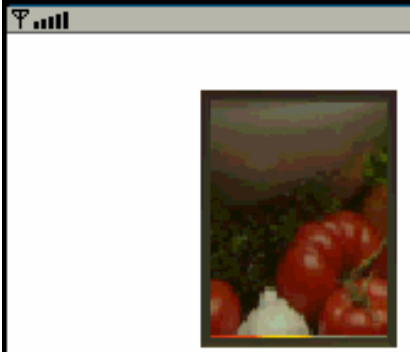

Obra fictícia para demonstraçẫo do museum. Nesta tela devern ser apresentadas informaçốes sobre a obra selecionada, utilizando imagem e texto. As informaçốes devern ser resumidas e recomenda-se apresentar uma referência para o usuário obter maiores detalhes.

Sair Voltar

Figura 2. a) Uma visão ampla de uma das salas do museu virtual. b) Uma visão aproximada da escultura virtual, destacando o menu com suas possibilidades de interação. c) A Interface de Conteúdo, apresentando as informações da obra selecionada. 


\section{Conclusões e Trabalhos Futuros}

Este trabalho mostrou o emprego das tecnologias de computação móvel e realidade virtual na educação, e apresentou um framework que reúne funcionalidades de ambas tecnologias, o VirTraM. Este consiste em uma arquitetura reutilizável e expansível para a construção de aplicações educacionais em dispositivos móveis. O protótipo desenvolvido, baseado no VirTraM, demonstrou a integração, de forma segmentada, entre o ambiente tridimensional e o conteúdo educativo, sem complicar a usabilidade da aplicação e considerando as restrições do visor do dispositivo. O protótipo também apresentou formas alternativas de interação, que permitiram ao usuário poder ora navegar ora manipular os objetos, interagindo apenas com as teclas do telefone celular. Por estas razões, o VirTraM pode evoluir e ser aplicado em outras áreas tais como: educação industrial, laboratórios de ciências e manutenção em campo.

Como trabalho futuro, planeja-se possibilitar, através da colaboração ou competição entre os visitantes, uma aprendizagem em grupo, utilizando-se tecnologias de comunicação sem fio. Outro plano para o futuro é a utilização de localização automática através de tecnologias como GPS (Global Positioning System) ou Wi-Fi, semelhante ao projeto desenvolvido por Göth et al. (2004), que utiliza essas técnicas para auxiliar um passeio em um campus universitário. Assim, a navegação do usuário dentro do ambiente virtual se daria de forma automatizada, à medida que ele se desloca no ambiente real.

\section{Referências Bibliográficas}

AHONEN, M.; JOYCE, B.; LEINO, M.; TURUNEN, H. Mobile Learning - A Different Viewpoint, In KYNÄSLAHTI, H.; SEPPÄLÄ, P. (Ed). Professional Mobile Learning. Helsinki: IT Press, 2003.

AVELLIS, G.; SCARAMUZZI, A.; FINKELSTEIN, A. Evaluating non-functional requirements in mobile learning contents and multimedia educational software. In: EUROPEAN CONFERENCE ON MOBILE LEARNING - MLEARN, 2003.

BRICKEN, M. Summer Students in Virtual Reality. WEXELBLAT, A. (Ed).Virtual Reality: Applications and Explorations. New York: Academic Press Professional, 1993.

CHARITOS, D.; LEPOURAS, G.; VASSILAKIS, C. Designing a virtual museum within a museum. In: CONFERENCE ON VIRTUAL REALITY, ARCHEOLOGY, AND CULTURAL HERITAGE, 2001.

CORCORAN, F.; DEMAINE, J.; DICAIRE, L.; PICARD, M.; TAYLOR J. Inuit 3D: An Interactive Virtual 3D Web Exhibition. In: MUSEUMS AND THE WEB CONFERENCE, 2002.

CRAWFORD, V.; VAHEY, P. Palm Education Pioneers Program. March, 2002 Evaluation Report. SRI International, Estados Unidos.

DANESH A.; INKPEN K.; LAU F., SHU K.; BOOTH K. Geney: designing a collaborative activity for the Palm handheld computer. In: CONFERENCE ON HUMAN FACTORS IN COMPUTING SYSTEMS, 2001.

ERICKSON, T. Artificial Realities as Data Visualization Environments. WEXELBLAT, A. (Ed). Virtual Reality: Applications and Explorations. New York: Academic Press Professional, 1993. 
FAYAD, M.; SCHMIDT, C.; JOHNSON, R. Building Application Frameworks: Object Oriented Foundations of Framework Design. 1999.

FERREIRA L.; TAROUCO L.; BECKER F. Fazer e compreender na Realidade Virtual: em busca de alternativas para o sujeito da aprendizagem. RENOTE - Revista Novas Tecnologias na Educação, Porto Alegre, 2004.

GÖTH, C.; HÄSS,U.-P.; SCHWABE, G. Requirements for mobile learning games shown on a mobile game prototype. In: EUROPEAN CONFERENCE ON MOBILE LEARNING - MLEARN, 2004.

KEEGAN, D. The future of learning: From e-learning to m-learning. Disponível em: <http://learning.ericsson.net/mlearning2/project_one/book.html>. Acesso em 15 dez. 2004.

KIRNER, C. Sistemas de Realidade Virtual. Disponível em: <http://www.dcc.ufscar.br/ grv>. Acesso em 15 dez. 2004.

LEHNER, F.; NÖSEKABEL, H. The Role Of Mobile Devices In E-Learning — First Experiences With A Wireless E-Learning Environment. In: IEEE INTERNATIONAL WORKSHOP ON WIRELESS AND MOBILE TECHNOLOGIES IN EDUCATION - WMTE, 2002.

LUCHINI, K.; QUINTANA C.; SOLOWAY E. Design Guidelines For LearnerCentered Handheld Tools. In: PROCEEDINGS OF THE 2004 CONFERENCE ON HUMAN FACTORS IN COMPUTING SYSTEMS, 2004.

NYIRI, K. Towards a philosophy of m-Learning. In: IEEE INTERNATIONAL WORKSHOP ON WIRELESS AND MOBILE TECHNOLOGIES IN EDUCATION - WMTE, 2002.

MARSON, F.; JUNG, C.; MUSSE, S. Modelagem Procedural de Cidades Virtuais. In: SYMPOSIUM ON VIRTUAL REALITY, 2003

MATTSSON, M. Object-oriented Frameworks: A Survey of Methodological Issues. Licentiate Thesis. Department of Computer Science, Lund University. Sweden. Disponível em: <http://www.ipd.bth.se/michaelm/papers/ >. Acesso em: 15 dez. 2004.

MEIRELLES L.; TAROUCO L.; ALVES C. Telemática Aplicada à Aprendizagem com Mobilidade. RENOTE - Revista Novas Tecnologias na Educação, Porto Alegre, 2004.

MYERS, B. A.; BEIGL, M. Handheld Computing. IEEE Computer Magazine, 2003.

OOSTERHOLT, R.; KUSANO, M.; VRIES G. Interaction Design Personal Communicator For Children. In: CONFERENCE ON HUMAN FACTORS IN COMPUTING SYSTEMS, 1996

ROUSSOU, M. Learning by doing and learning through play: an exploration of interactivity in virtual environments for children. Computers in Entertainment - CIE, 2004.

SUN. Java 2 Platform, Micro Edition (J2ME). Disponível em: <http://java.sun.com/j2me/index.jsp>. Acesso em 15 dez. 2004.

SYVÄNEN, A.; AHONEN, M.; JÄPPINEN, A.; PEHKONEN, M.; VAINIO, T. Accessibility And Mobile Learning. In: IFIP ETRAIN CONFERENCE IN PORI, Finland, 2003. 
$-\int$ CINTED-UFRGS Novas Tecnologias na Educação

WAZLAWICK, S. Collaborative Learning In A Virtual Museum Authoring Tool. In: SYMPOSIUM ON VIRTUAL REALITY, 2001.

WOJCIECHOWSKI, R.; WALCZAK K.; WHITE M.; CELLARY W. Production: Building Virtual And Augmented Reality Museum Exhibitions. In: THE NINTH INTERNATIONAL CONFERENCE ON 3D WEB TECHNOLOGY, 2004 